$$
6
$$




\section{DISCLAIMER}

This report was prepared as an account of work sponsored by an agency of the United States Government. Neither the United States Government nor any agency Thereof, nor any of their employees, makes any warranty, express or implied, or assumes any legal liability or responsibility for the accuracy, completeness, or usefulness of any information, apparatus, product, or process disclosed, or represents that its use would not infringe privately owned rights. Reference herein to any specific commercial product, process, or service by trade name, trademark, manufacturer, or otherwise does not necessarily constitute or imply its endorsement, recommendation, or favoring by the United States Government or any agency thereof. The views and opinions of authors expressed herein do not necessarily state or reflect those of the United States Government or any agency thereof. 


\section{DISCLAIMER}

Portions of this document may be illegible in electronic image products. Images are produced from the best available original document. 
This report was prepared as an account of Government sponsored work. Neither the United states, nor the Commission, nor any person acting on behalf of the Commission:

A. Makes any warranty or representation, expressed or implied, with respect to the accuracy, completeness, or usefulness of the information contained in this report, or that the use of any information, apparatus, method, or process disclosed in this report may not infringe privately owned rights; or $1, y$

B. Assumes any liabilities with respect to the use of, or for damages resulting from the use of any information, apparatus, method, or process disclosed in this report.

As used in the above. "person acting on behalf of the Commlssion" Includes any employee or contractor of the Commission, or employee of such contractor, to the extent that such employee or contractor of the Commission, or employee of such contractor prepares, diseeminates, or provides access to, any information pursuant to his employment or contract with the Commission, or his employment with such contractor.

Printed in USA. Price $\$ 2$. Available from the Clearinghouse for Federal Scientific and Technical Information, National Bureau of Standards,

U. S. Department of Commerce, Springfield, Virginia 
C. 2.60 . IAN 50

RELEASED FOR ANNOUNCEMENT

IN NUCLEAR SCIENCE

\title{
ELLIPSOMETER STUDIES ON THE CLEANING AND OXIDATION OF URANIUM
}

\author{
by
}

D. T. Larson

N.W. Taylor

\begin{abstract}
LEGAL NOTICE
This report was prepared as an account of Government sponsored work. Neither the United States, nor the Commission, nor any person acting on behalf of the Commission:

A. Makes any warranty or representation, expressed or implied, with respect to the accuracy, completeness, or usefulness of the information contained in this report, or that the use of eny information, apparatus, method, or process disclosed in this report may not infringe privately owned rights; or

B. Assumes any liabilities with respect to the use of, or for damages resulting from the use of any Information, apparatus, method, or process disclosed in this report.

As used in the above, "person acting on behalf of the Commission" includes any employee or contractor of the Commission, or employee of such contractor, to the extent that such employee or contractor of the Commission, or employee of such contractor prepares, disseminates, or provides access to, any information pursuant to his employment or contract with the Commission, or his employment with such contractor.
\end{abstract}

\section{THE DOW CHEMICAL COMPANY \\ ROCKY FLATS DIVISION \\ P. O. BOX 888 \\ GOLDEN, COLORADO 80401}

U.S. ATOMIC ENERGY COMMISSION

CONTRACT AT(29-1)-1106 


\section{ACKNOWLEDGMENTS}

The authors wish to express their appreciation to Car1 A. Larson, who prepared the computer program necessary for evaluation of ellipsometer data, and to Robert $J$. Bouchard, who lapped the uranium coupons. 
CONTENTS

Page No.

Acknowledgments . . . . . . . . . . . . . . . . . ii

Abstract ......................... iv

Summary and Conclusions . . . . . . . . . . . . . . . v v

Introduction ......................... 1

Experimental ........................... . . . 2

Apparatus . . . . . . . . . . . . . . . . . . 2

Procedure ......................... 4

Results and Discussion .................. . . 6

Determination of Complex Index of Refraction for Uranium ....................... 6

Analysis of the Oxidation of Uranium at 50 Microns

Oxygen and $230 \mathrm{C}$ Using the Linear Equations . . . . . . . 8

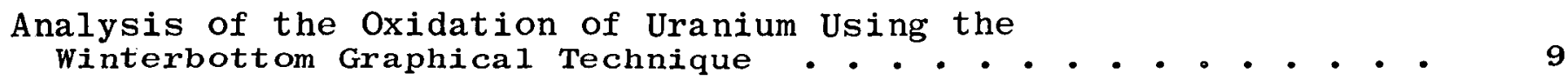

Appendix: Analysis of Ellipsometer Data . . . . . . . . . 13 


\begin{abstract}
An investigation was conducted (with an ellipsometer) on the feasibility of studying gas reaction kinetics and cathodic sputter cleaning of uranium. A laboratory ellipsometer was constructed, and isothermal oxidation and cathodic sputter cleaning were investigated.
\end{abstract}




\section{SUMMARY AND CONCLUSIONS}

An ellipsometer has been found to be an effective instrument in the investigation of sputter cleaned and oxidized uranium. The experimental and theoretical equations, necessary for the determination of the optical constants of a clean surface and the index of refraction and thickness of films on the surface, have been solved algebraically and the solutions programmed for a computer.

Uranium coupons were sputter cleaned in argon, and ellipsometer readings were taken until no further change was noted. A reproducible value of the endpoint of the sputter cleaning process was obtained for several different samples which was assumed to represent the value of a clean surface. From this, the index of refraction of sputter cleaned uranium was calculated to be $3.10-3.06 i$. The results demonstrated the suitability of an ellipsometer for measuring a reproducible state of the surface as a starting point for gas-metal reaction studies.

Oxide film thickness and the index of refraction were determined using the Winterbottom graphical technique. The index of refraction of the oxide films was found to be 2.1 - 0.5i. This index of refraction for oxides prepared at 50 microns oxygen and $23^{\circ}, 50^{\circ}$ and $75^{\circ} \mathrm{C}$ was the same, although the index of refraction of the oxide prepared at 50 microns oxygen and $100 \circ \mathrm{C}$ appeared to be slightly different. Oxide thicknesses as a function of time were also obtained for each of the oxidation temperatures; $23^{\circ}, 50^{\circ}$ and $100^{\circ} \mathrm{C}$. 
RFP-674

$v i$ 


\section{INTRODUCTION}

An ellipsometer can be used to measure the index of refraction of a clean metal surface and the index of refraction and thickness $(1,2,3)$ of an adsorbed film. The ellipsometer measures the statistical or average thickness of the thin film on the metal surface because the interface of the metal surface and the thin film may not be well-defined, and the film thickness may be irregular because of different rates of film growth. The ellipsometer is used to follow surface reaction kinetics; also, it can be used to determine the state of cleanliness of the surface.

The advantages of an ellipsometer in making surface studies, according to J. V. Cathcart and G. F. Petersen, (4) are:

- The nondestructive nature of the measurements

- The possibility of making essentially continuous measurements at a distance

- The extreme sensitivity of the method.

U. R. Evans (5) reports that an ellipsometer provides a useful technique for determining thickness of films on a surface, although the conversion of ellipsometer measurements to film thickness is a tedious procedure. Despite this difficulty, the advantages in the study of surfaces with an ellipsometer appear to outweigh this disadvantage.

The experimental investigation was divided into two parts. The first was a study of the preparation of a sputter cleaned uranium surface and determination of the optical constants. The second was a study of the oxidation kinetics of uranium.

(1) Menard, R. C., J. Opt. Soc. Am., 52, 427 (1962).

(2) Archer, R. J., J. Opt. Soc. Am., 52,970 (1962).

(3) McCrackin, F. L., Passaglia, E., Stromberg, R. R., and Steinberg, H. L., J. Res. Nat1. Bur. Std. (U.S.), 67A, 363 (1963).

(4) Cathcart, J. V., and Petersen, G. F., Ellipsometry in the Measurement of Surfaces and Thin Films, E. Passaglia, R. R. Stromberg, and

J. Kruger, ed., Washington, D.C., Nat1. Bur. Std., 1964, Miscellaneous Publication 256, p 201 .

(5) Evans, U. R., The Corrosion and Oxidation of Metals, London, Arnold, 1961, p 790 . 
EXPER IMENTAL

\section{Apparatus}

The experimental apparatus is shown in Figure 1. The apparatus consisted of two main parts: the ellipsometer and the high vacuum system. The high vacuum was achieved with a 40 liter a second sputter ion pump. After bakeout, typical pressures were in the low 10-7 torr range in the sample chamber. The sample chamber (Figure 2) was a modified 4-in. glass adapter which was closed and fitted with Pyrex windows. The windows were annealed to remove strains so that the state of polarization of the light entering and leaving the glass chamber was not changed. A copper-constantan thermocouple and a negative potential lead for cathodic sputtering were attached to the sample holder.

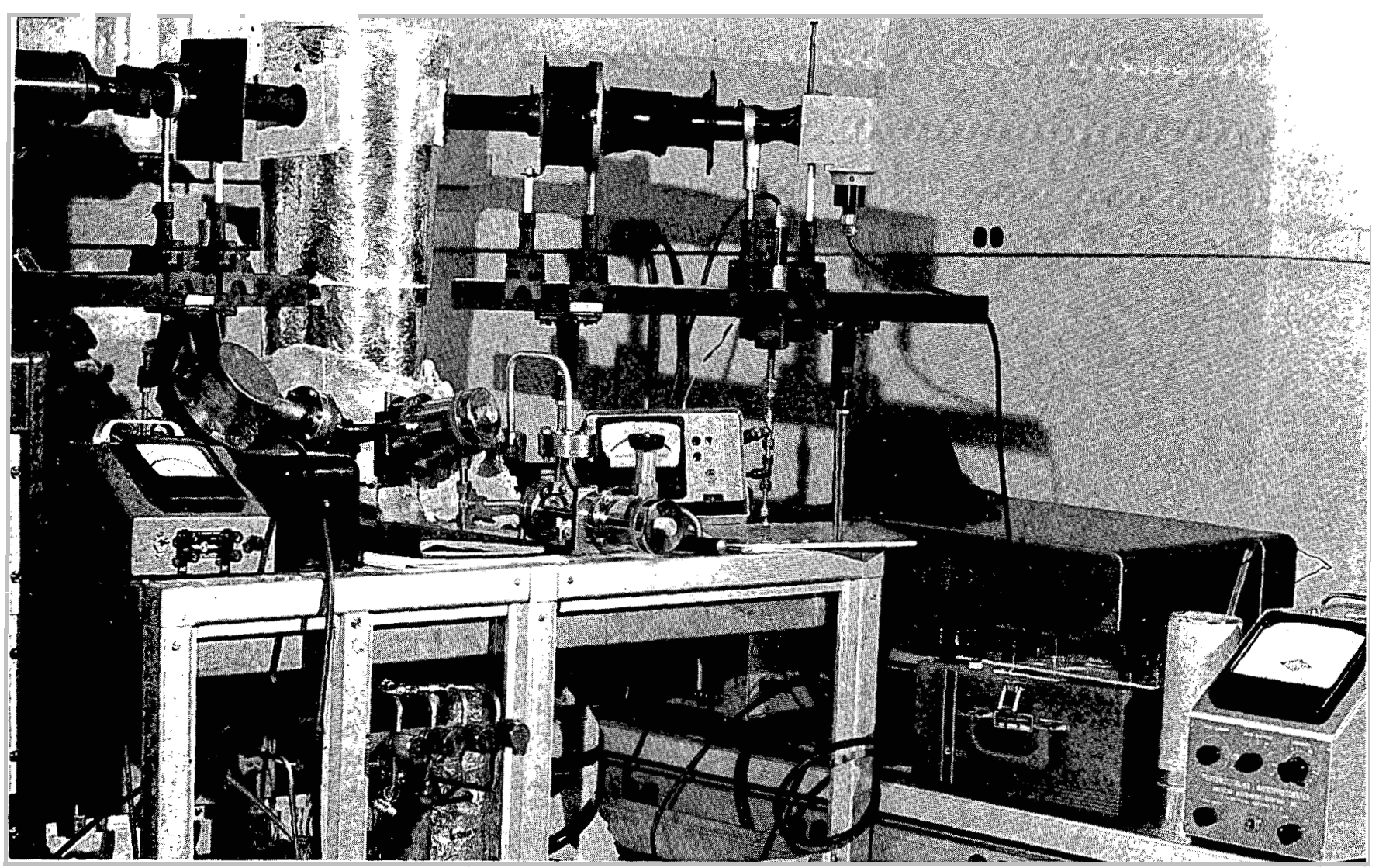

Figure 1. ELLIPSOMETER WITH HIGH VACUUM SYSTEM 


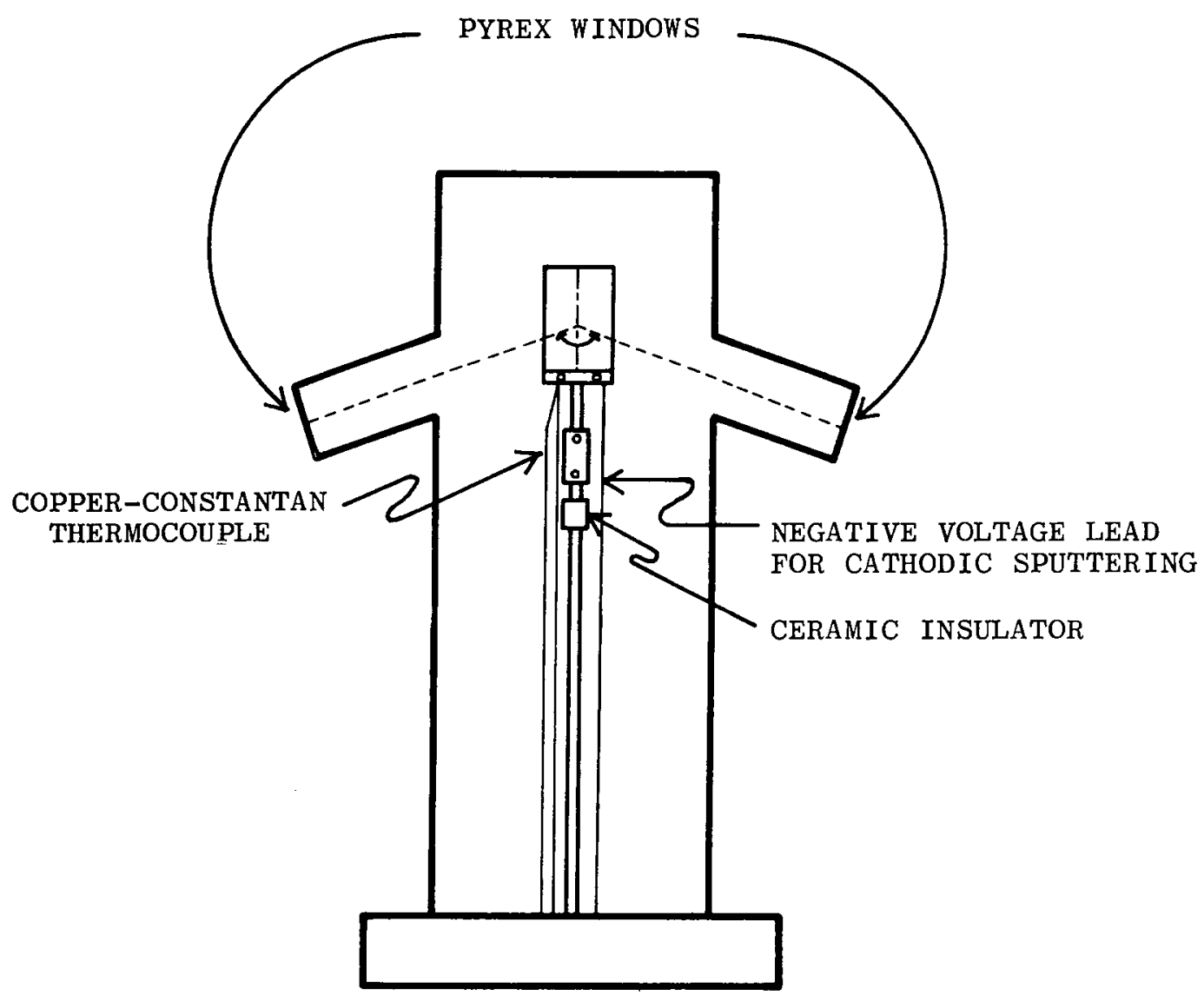

Figure 2. SAMPLE CHAMBER

The special features of the vacuum system included a pure gas injection manifold, pressure and temperature control, and provision for cathodic sputter cleaning. The manufacturer's specifications for the purity of the research grade argon was $99.9995 \mathrm{~min}$. vol. percent, and the oxygen was 99.95 min. vol. percent.

The schematic of the ellipsometer optical system is shown in Figure 3 . The light source was a mercury arc lamp used with a filter to obtain monochromatic light at $5461 \AA$; the light beam was collimated with iris diaphragms. The polarizer and analyzer were Nicol prisms. Monochromatic light was plane polarized at $45^{\circ}$ to the plane of incidence. After reflection from the metal sample, the plane polarized light became elliptically polarized. The quarter-wave plate and analyzer were used to determine the parameters of this reflected, elliptically polarized light. The elliptically polarized ght then passed through the quarter-wave plate which, when properly oriented, reestablished the plane polarized light. The plane polarized light 
RFP-674

then passed through the analyzer, which, when properly oriented extinguished the light. The extinction position was determined by a photomultiplier microphotometer.

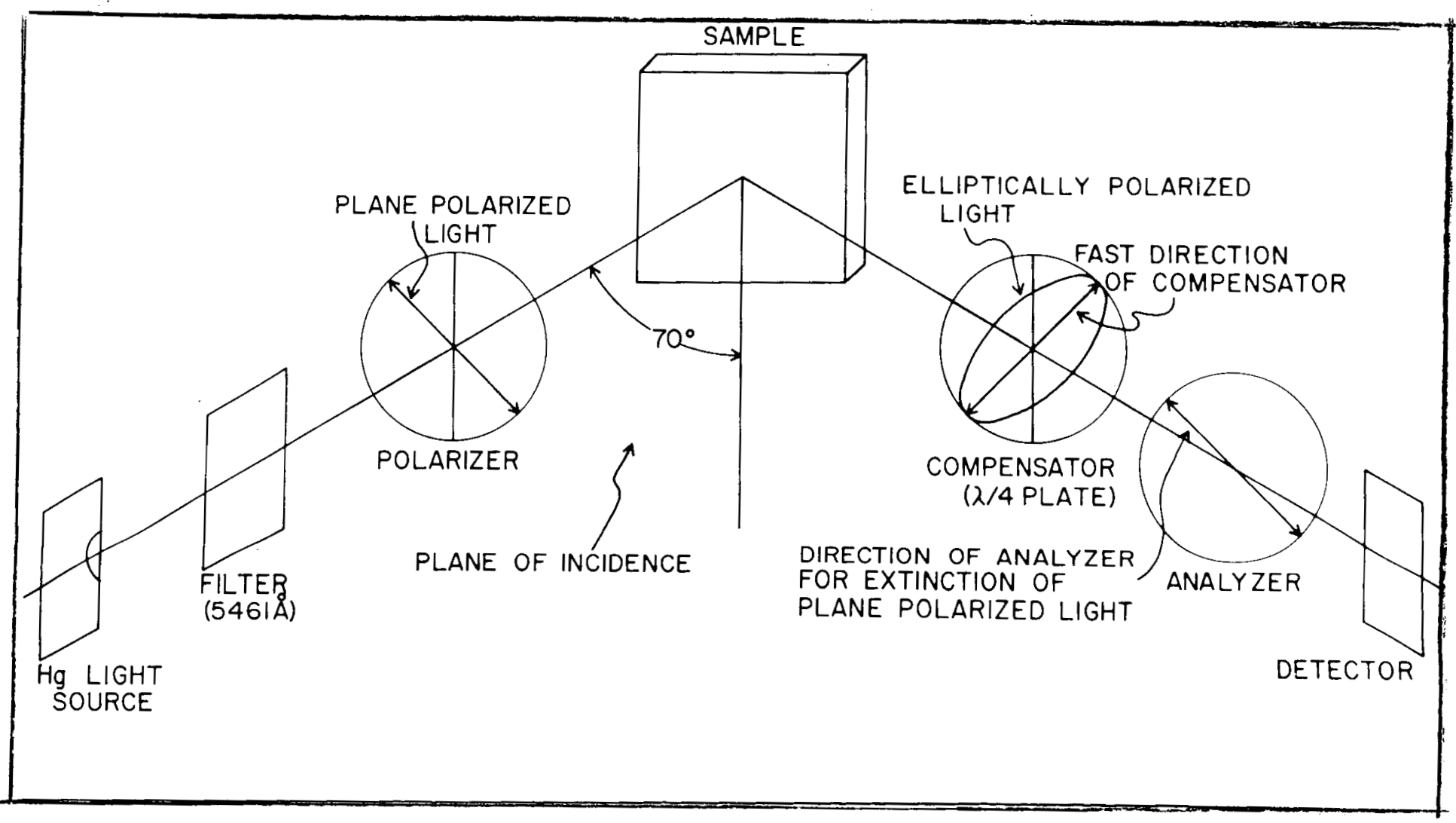

10751

Figure 3. ELLIPSOMETER OPTICAL SYSTEM

Procedure

The experimental procedure involved placing the metal sample in the vacuum chamber, cleaning by cathodic sputtering in argon, and oxidizing by exposure to oxygen at various pressures and temperatures. The changes in the state of the metal surface were followed in situ with the ellipsometer. The metal samples were prepared by lapping and polishing uranium coupons ( $1-15 / 16$ in. by $1-7 / 16$ in. by $1 / 16$ in.) in a slurry of aluminum oxide and kerosene. The 
aluminum oxide was 1900 grit and the surface finish was 7 to 8 microinches. The sample was hand polished immediately prior to being placed in the vacuum lamber; the polish used was Elgin diamond abrasive, grade 3 initially and grade 1 on later samples.

After properly aligning the sample so that a maximum intensity was obtained at the photomultiplier when the angle of incidence was 70.0 degrees, the chamber was evacuated and baked at $250^{\circ} \mathrm{C}$ for several hours. Upon cooling, the sample was cathodically sputtered in argon until no further change in ellipsometer readings was noted. Oxidation at various pressures and temperatures was followed with the ellipsometer. The angular orientations of the quarter-wave plate and the analyzer were determined at extinction of the light.

The extinction position was found by taking increments in the quarter-wave plate readings and adjusting the analyzer to obtain a relative minimum intensity. A graph of quarter-wave settings versus intensity determined the quarter-wave value for an absolute intensity minimum; the associated value of the analyzer thus gave the two readings for the extinction position. This method of determining the extinction position is discussed in detail by Mertens, Theroux, and Plumb, ${ }^{(6)}$ and it insures a greater accuracy in finding the extinction position than when the quarter-wave plate and analyzer are rotated simultaneously.

From the angular orientations of the quarter-wave plate and analyzer at the extinction position, two parameters describing the state of polarization of the elliptically polarized light may be determined. These are the $\Psi$ and $\Delta$ values where $\Psi$ is defined as the angle whose tangent is the ratio of the amplitudes of the parallel and normal components of the reflected polarized light and $\Delta$ is the phase difference. The parameters $\Delta^{\prime}$ and $\Psi^{\prime}$ represent a clean surface. Our definition of a clean surface is the state of the uranium surface after repeated argon bombardment produces no further change in ellipsometer measurements.

6) Mertens, F. P., Theroux, P., and Plumb, R. C., J. Opt. Soc. Am., 53, 788 (1963). 
RFP-674

The values of $\Delta^{\prime}$ and $\Psi^{\prime}$ are used to determine the complex index of refraction of uranium, and values of $\Delta$ and $\Psi$ are used to determine the index of refraction and thickness of films on the uranium surface. The details of the procedure are to be found in the Appendix.

Calculations were made using an ASI-210 computer.

\section{RESULTS AND DISCUSSION}

Determination of Complex Index of Refraction for Uranium

To measure the complex index of refraction, it is necessary to measure the $\Delta^{\prime}$ and $\Psi^{8}$ values representative of a clean surface. The method used to prepare a clean surface was cathodic sputtering in argon. Table I shows various values of $\Delta^{\prime}$ and $\Psi^{\prime}$ for different samples with clean surfaces prepared at different sputtering voltages and an argon pressure of $45 \mathrm{microns}$. The samples were sputtered until no change was noted in ellipsometer readings. It is interesting to note that the $\Delta^{\prime}$ and $\Psi^{\prime}$ values for different samples agree reasonably well, thus providing a starting point for gas metal reaction studies.

TABLE I

VALUES OF $\Delta^{\prime}$ AND $\Psi^{\prime}$ FOR DIFFERENT SAMPLES PREPARED BY CATHODIC SPUTTERING IN ARGON AT VARIOUS SPUTTERING VOLTAGES

\begin{tabular}{|c|c|c|c|c|c|}
\hline $\begin{array}{l}\text { Sample } \\
\text { Number }\end{array}$ & $\begin{array}{c}\text { Sputtering } \\
\text { Voltage (volts) }\end{array}$ & $\begin{array}{c}\text { Current Density } \\
\mathrm{ma} / \mathrm{cm}^{2}\end{array}$ & $\begin{array}{c}\text { Argon Pressure } \\
\text { microns } \\
\end{array}$ & $\begin{array}{c}\Delta^{\prime} \\
\text { degrees } \\
\end{array}$ & $\begin{array}{c}\Psi^{\prime} \\
\text { degrees }\end{array}$ \\
\hline 1 & 750 & 0.038 & 45 & 122.4 & 27.1 \\
\hline 2 & 1000 & 0.093 & 45 & 124.1 & 27.0 \\
\hline 3 & 1250 & 0.15 & 45 & 126.5 & 25.4 \\
\hline 4 & 1500 & 0.27 & 45 & 125.4 & 26.1 \\
\hline 5 & 1750 & 0.42 & 45 & 125.1 & 26.7 \\
\hline 6 & 2000 & 0.48 & 45 & 127.3 & 26.2 \\
\hline
\end{tabular}

The values selected as representative of a clean surface are $\Delta^{\prime}=127.3^{\circ}$ and $\Psi^{\prime}=26.2^{\circ}$. As noted by experimental cleaning (Figure 4) and oxidation curves of $\Delta$ and $\Psi$, one finds that $\Delta$ increases as film thickness decreases while $\Psi$ varies by only a small amount near the clean surface. Table I 


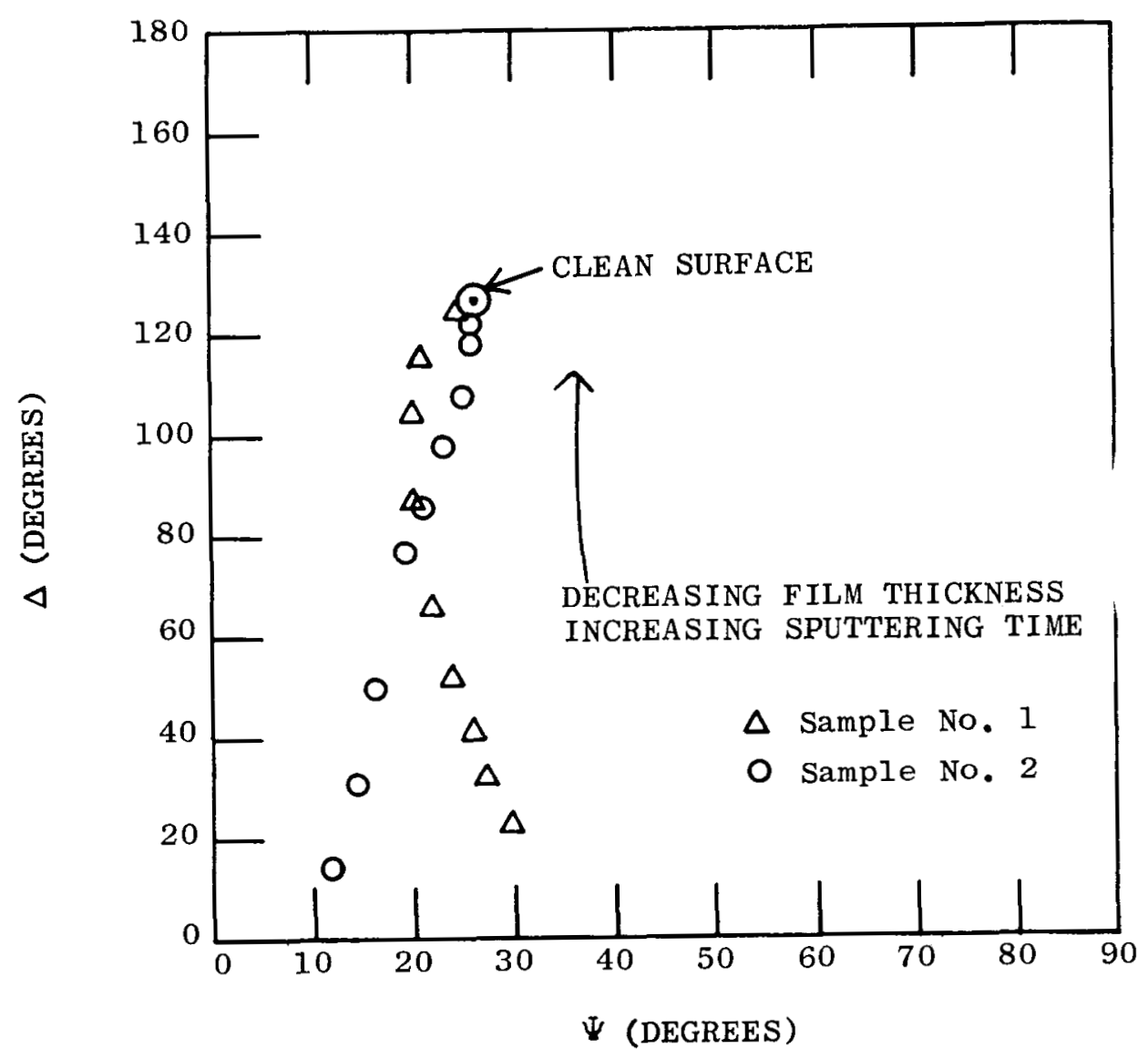

Figure 4. CATHODIC SPUTTER CLEANING CURVES ( $\Delta$ versus $\Psi$ ) AT 2000 VOLTS AND 45 MICRONS ARGON

shows that the greatest value of $\Delta^{\prime}$ is $127.3^{\circ}$, and consequently this was taken as the best value for $\Delta^{\prime}$ for a clean surface. Because of the difficulties in obtaining a film free surface, the values of $\Delta^{\prime}$ and $\Psi^{\prime}$ are not exact and contain a certain unavoidable experimental error. There is no guarantee that sputter cleaning produces a film free surface; indeed, it is quite possible that some type of oxide film exists on the surface after the sputtering, but because of the reproducibility of the sputter endpoints, the above values of $\Delta^{\prime}$ and $\Psi^{\prime}$ are taken as the definition of a clean uranium surface.

Using these values for $\Delta^{\prime}$ and $\Psi^{\prime}\left(\Delta^{\prime}=127.3^{\circ} ; \Psi^{\prime}=26.2^{\circ}\right)$, the index of refraction for a film free uranium substrate ( $\hat{n}_{3}=n_{3_{1}}-n_{32} i$ ) was alculated by the equations in the Appendix to be $\hat{n}_{a}=3.10-3.06 i$. 


\section{RFP-674}

Analysis of the Oxidation of Uranium at 50 Microns Oxygen and $23^{\circ} \mathrm{C}$ Using the I,inear Equations

For the oxidation of uranium at room temperature the parameters $\Delta$ and $\Psi$ did not change enough to obtain a well defined curve to which a theoretical curve could be fitted; therefore, the measurement of the complex index of refraction of the oxide film by Winterbottom's graphical method was precluded. Instead of using the graphical technique, an approximation to the exact equations was used which assumes the film on the metal substrate to be nonabsorbing, and consequently to possess an index of refraction that is real. The approximate equations have algebraic solutions which give good thickness results for films not greater than 100 angstroms. The equations used which present fewer approximations than the original Drude linear equations are presented by Lucy ${ }^{(7)}$ where two equations relating the dielectric constant $\epsilon$ and the thickness of the film are presented. The results of the calculations from these equations for the oxidation of uranium at room temperature, $23^{\circ} \mathrm{C}$, and 50 microns oxygen are shown in Figure 5 .

(7) Lucy, F. A., J. Chem. Phys., 16, 167 (1948).

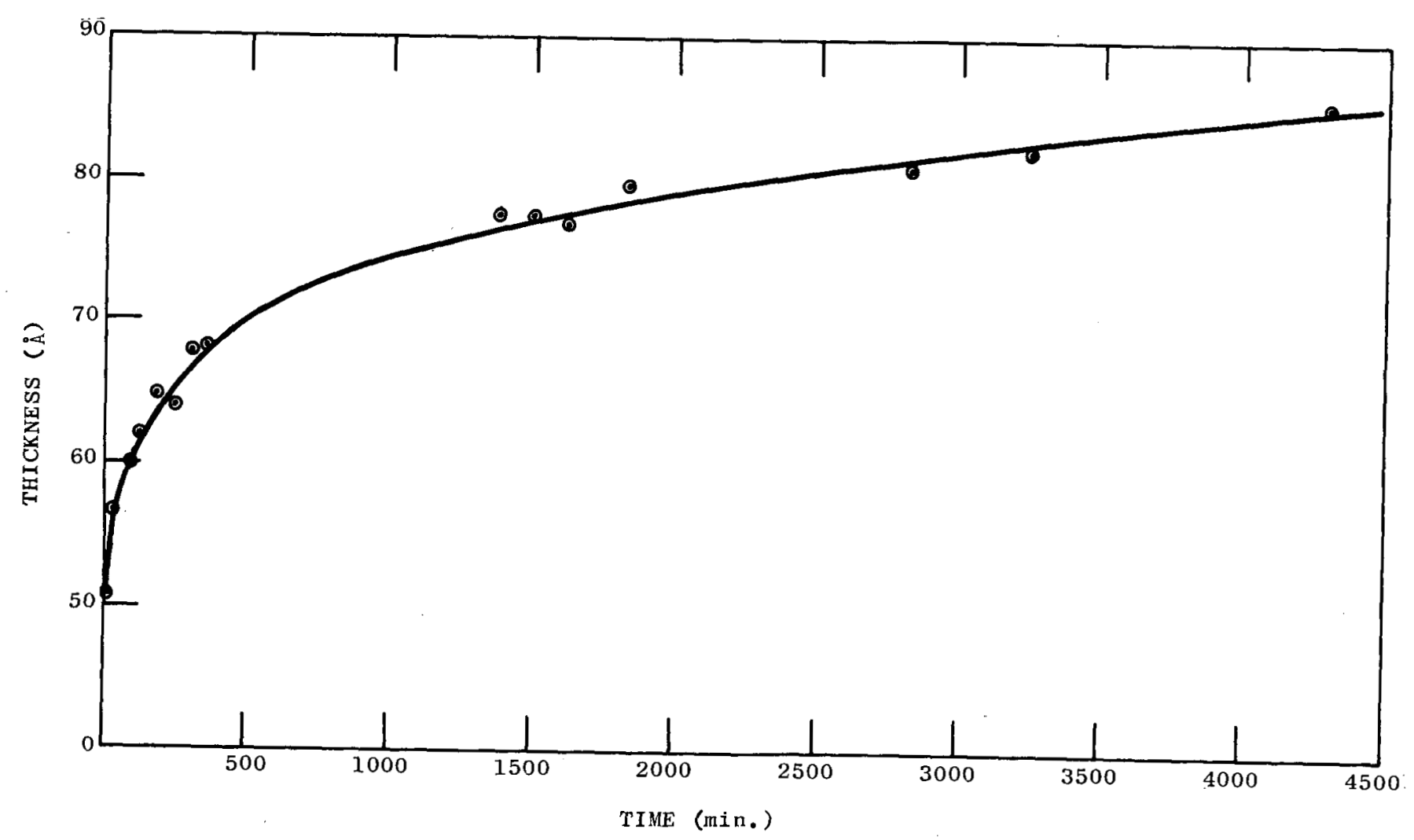

Figure 5. THICKNESS AS A FINCTION OF TIME FOR URANIUM OXIDATION AT 50 MICRONS OXYGEN AT $23^{\circ} \mathrm{C}$ 
The index of refraction as well as the thickness for each data point had to ie calculated. The $\Delta^{\prime}$ and $\Psi^{\prime}$ values for a clean surface and the corresponding values for the optical constants of the base metal substrate were used in the calculation. The calculated values of the dielectric constant of the oxide film were not constant but varied from 16.2 to 24.8. The index of refraction is equal to the square root of the dielectric constant. These values seem high by a factor of two, but this is not unexpected. Peterson and Bashara ${ }^{(8)}$ report that the error in the calculated real refraction index of an absorbing dielectric film which is determined by approximate equations that neglect absorption, can be greater than 100 percent. The error in the thickness determinations is considerably less since the dielectric constant enters into the thickness calculation by a factor of $(\epsilon-1) / \epsilon$. Thus, we may have a greater reliance on the computed values of thickness than on the dielectric constant.

Analysis of the Oxidation of Uranium Using the Winterbottom Graphical Technique

The experimental $\Delta$ versus $\Psi$ points for oxidation at 50 microns oxygen and $23^{\circ}, 50^{\circ}$ and $75^{\circ} \mathrm{C}$ appeared to lay on the same curve. However, the experimental points for oxidation at 50 microns oxygen and $100^{\circ} \mathrm{C}$ varied slightly from this curve (Figure 6). Since each theoretical curve is calculated for one index of refraction of the film, this variation of the curve at $100^{\circ} \mathrm{C}$ could be attributed to a change of the index of refraction of the oxide film formed at $1000^{\circ} \mathrm{C}$. More data would have to be analyzed for a quantitative determination.

Figure 7 shows the matching of a theoretical curve with the experimental oxidation points at 50 microns oxygen and $75^{\circ} \mathrm{C}$. A family of theoretical curves was generated over a film thickness range by changing the index of refraction of the film both real and imaginary parts. The theoretical curve which gave the best fit to the experimental points was calculated with $\hat{n}_{2}=2.1-0.5 i$ which is concluded to be the index of refraction of the oxide film.

(8) Peterson, D. W., Bashara, N. M., J. Opt. Soc. Am., 55, 845 (1965). 
Figure 6. EXPERIMENTAL $\triangle$ VERSUS $\Psi$ CURVE FOR URANIUM OXIDATION AT 50 MICRONS OXYGEN AND $23^{\circ}$, $50^{\circ}, 75^{\circ}$, AND $100^{\circ} \mathrm{C}$

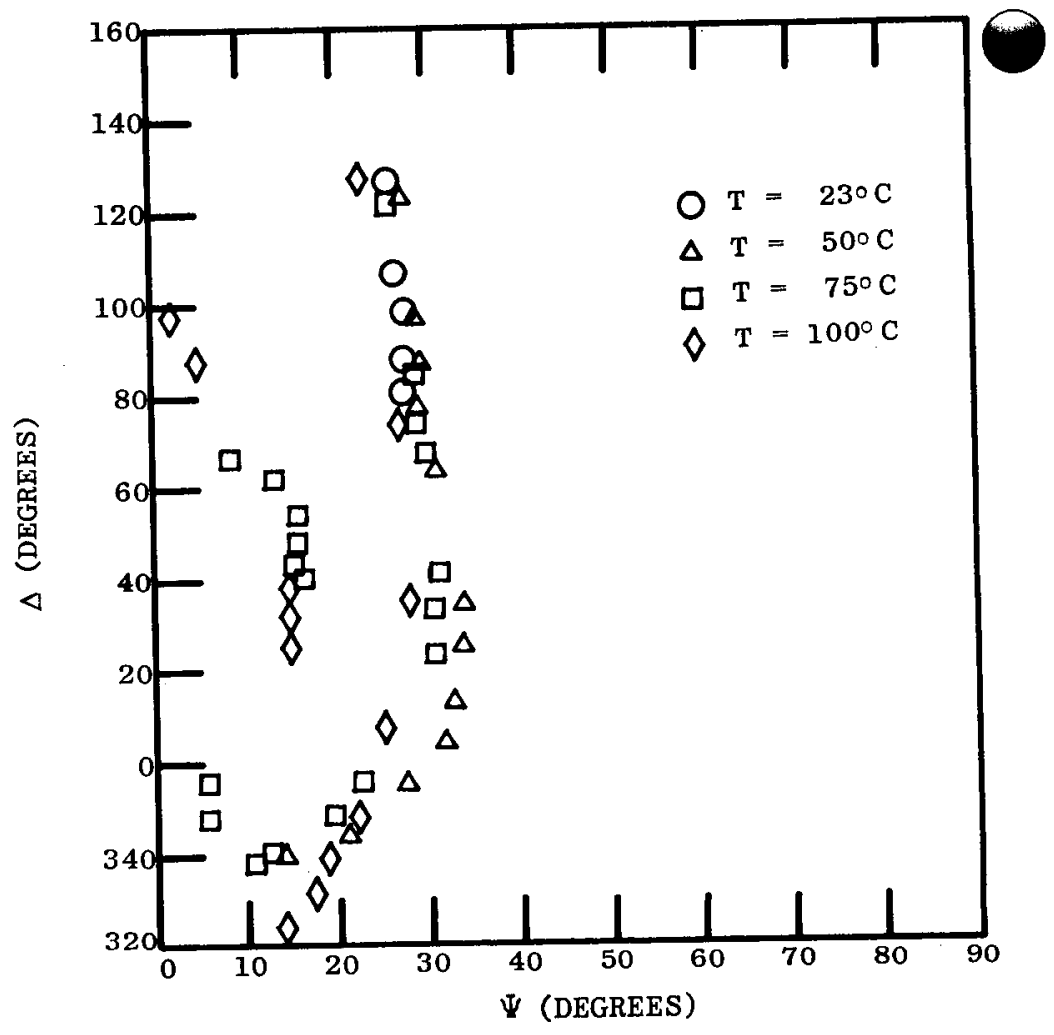

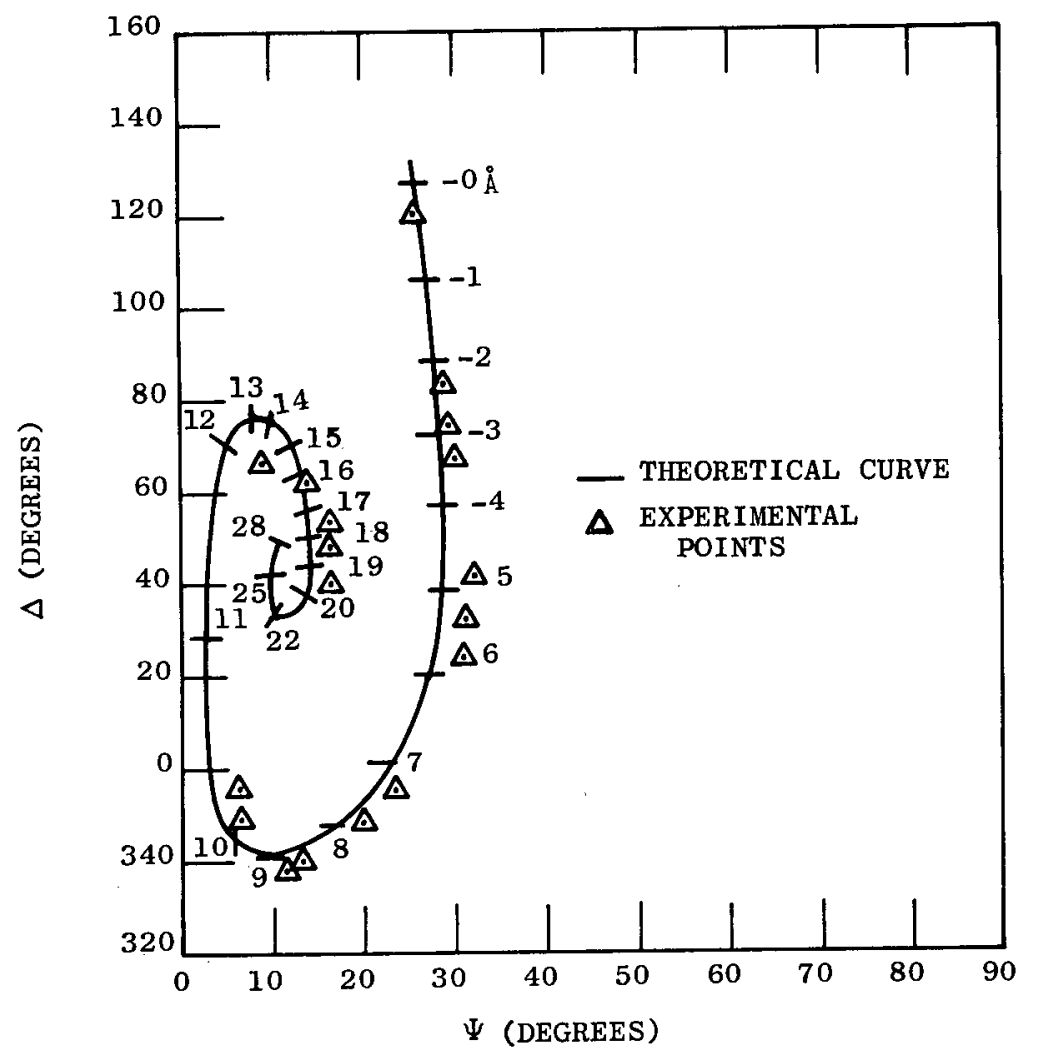

Figure 7. THEORETICAL $\Delta$ VERSUS $\Psi$ CURVE WITH $\hat{n}_{3}=3.10-3.06 \mathrm{i}$ AND $\hat{n}_{2}=2.1-$ $0.5 \mathrm{i}$ FOR FILM THICKNESS FROM 0 TO 2800A MATCHING EXPERIMENTAL $\triangle$ VERSUS $\Psi$ POINTS FOR URANIUM OXIDATION AT 50 MICRONS OXYGEN AND $75^{\circ} \mathrm{C}$. NUMBERS ON THE THEORETICAL CURVE INDICATE FILM THICKNESS IN HUNDREDS OF ANGSTROMS 
This theoretical curve, from 0 to $2800 \AA$, was used to determine film thickpesses at various points on the experimental oxidation curves. The results are shown in Figure 8 where film thickness is plotted as a function of time for the various oxidation temperatures. The oxidation curve at $23^{\circ} \mathrm{C}$ differs from the same oxidation curve in Figure 5 due to the different methods of calculating the film thickness. Thicknesses for Figure 5 were calculated using Lucy's method which uses approximations to the exact Drude equations and applies only to thin films less than $100 \AA$. However, thicknesses in the oxidation curve in Figure 8 were calculated using the Winterbottom graphical technique which employs the exact Drude equations. Thus, one would expect the calculated film thickness in Figure 8 to be closest to the true value.

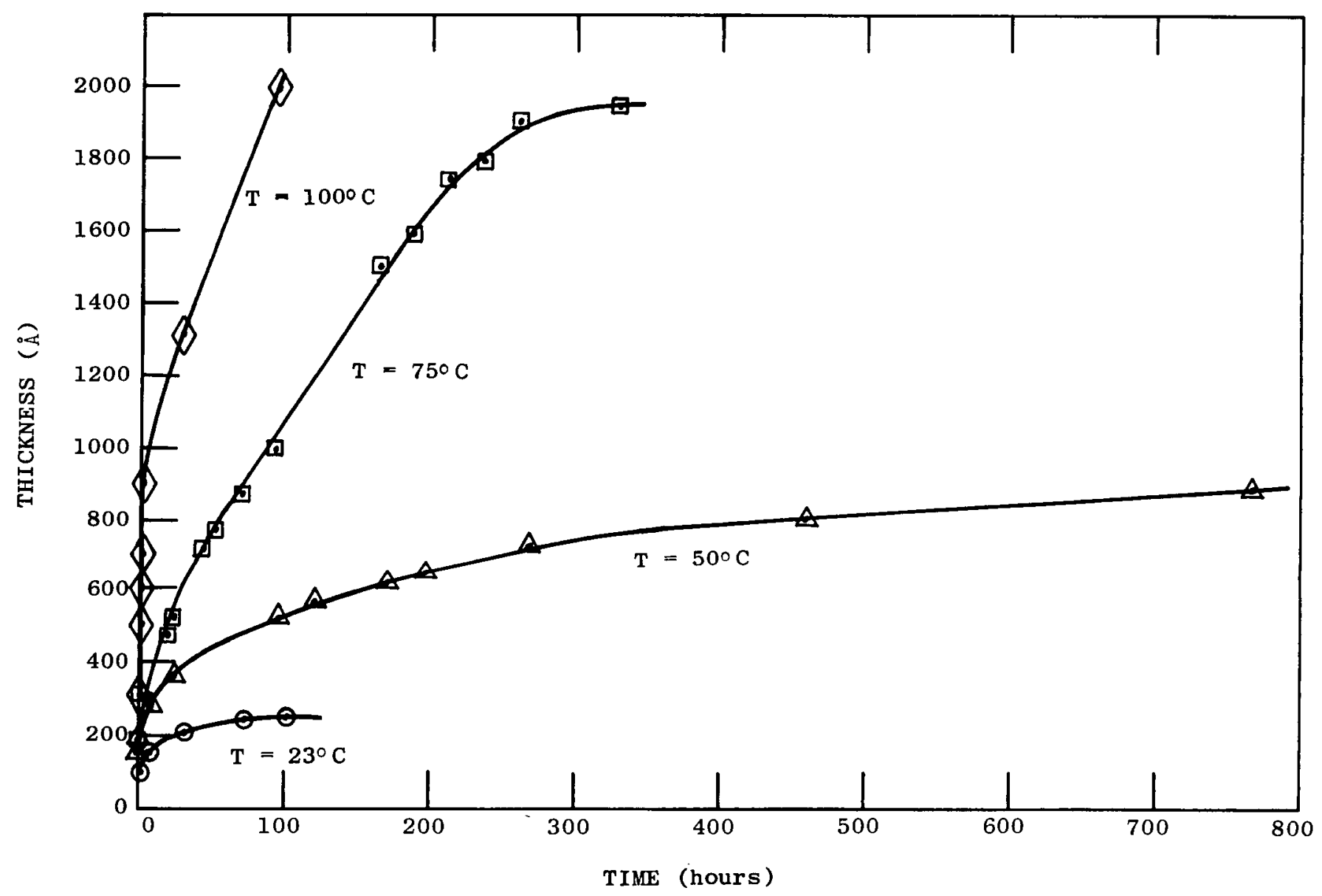

Figure 8. THICKNESS AS A FUNCTION OF TIME FOR URANIUM OXIDATION AT 50 MICRONS OXYGEN 


\section{RFP-674}

The following constants were used in the calculations:

- Intlex of refraction of surrounding gaseous medium, $n_{I}=1.0$

- Angle of incidence, $\varphi_{1}=70.0^{\circ}$

- Wavelength of light in vacuum, $\lambda=5461 \AA$

- Phase retardation of quarter-wave plate, $\omega=96.8^{\circ}$. 


\section{APPENDIX}

Analysis of Ellipsometer Data

One method of analyzing ellipsometer data is by Winterbottom's graphical technique. (1,2,9) A brief discussion of this technique, with a detailed explanation of the equations used, is given.

From ellipsometer measurements, the experimental $\Delta^{\prime}$ s and $\Psi^{\prime}$ s are determined, where $\Psi$ is defined as the angle whose tangent is the ratio of the amplitudes of the parallel and normal components of the reflected polarized light and $\Delta$ is the phase difference. A clean surface (free of surface films) is prepared and ellipsometer measurements are taken to determine a $\Psi^{\prime}$ and $\Delta^{\prime}$ which are representative of a clean surface. These quantities are used to determine the complex index of refraction for a clean surface. Next, assumed values for the index of refraction of the adsorbed film, either complex or real, and thicknesses of the film, are used to compute theoretical $\Psi^{\prime}$ s and $\Delta^{\prime} s$. A theoretical curve of $\Delta$ versus $\Psi$ is matched with the experimental curve of $\Delta$ versus $\Psi$, which enables the determination of the index of refraction and thicknesses of the film.

Experimental values of $\Psi$ and $\Delta$ are obtained from the following equations: (10)

$$
\begin{aligned}
& \cos 2 \Psi=\cos 2_{\gamma} \cos 2_{\alpha}\left(1-\cos \omega \tan 2_{\alpha} \tan 2_{\gamma}\right), \\
& \cos \Delta=\frac{\cos 2_{\gamma} \cos 2_{\alpha}\left(\tan 2_{\alpha}+\cos \omega \tan 2_{\gamma}\right)}{\sin 2_{\Psi}}
\end{aligned}
$$

$\omega$ is the phase retardation of quarter-wave plate calculated by the equation:

$$
\cos \omega=\frac{\cos 2_{\gamma}(1) \cos 2_{\alpha}(1)-\cos 2_{\gamma}(2) \cos 2_{\alpha}(2)}{\sin 2_{\gamma}(1) \sin 2_{\alpha}(1)-\sin 2_{\gamma}(2) \sin 2_{\alpha}(2)}
$$

(1) Menard, R. C., loc. cit., p 1.

(2) Archer, R. J., loc. cit., p 1.

(9) Theroux, P., Plumb, R. C., J. Opt. Soc. Am., 53, 1451 (1963).

(10) Plumb, R. C., J. Opt. Soc. Am., 50, 892 (1960). 
RFP-674

The angles $\alpha$ and $\gamma$ and their relationship to the elliptically polarized light are shown in Figure 9. The angle $\gamma$ is related to the angles $\alpha$ and $\beta$ by the relationship $\gamma=\beta-\alpha$. The angle of the quarter-wave plate is represented by $\alpha$, and $\beta$ is the angle of the analyzer, with respect to their zero azimuth positions. The zero azimuth was selected perpendicular to the plane of incidence. The arbitrary convention that all angles measured clockwise are negative and all angles measured counterclockwise are positive, looking toward the light source, was adopted. In Equation (3), the (1) and (2) stand for extinction of light in the two compensating positions of the quarter-wave plate; the fast, and the slow positions, respectively.

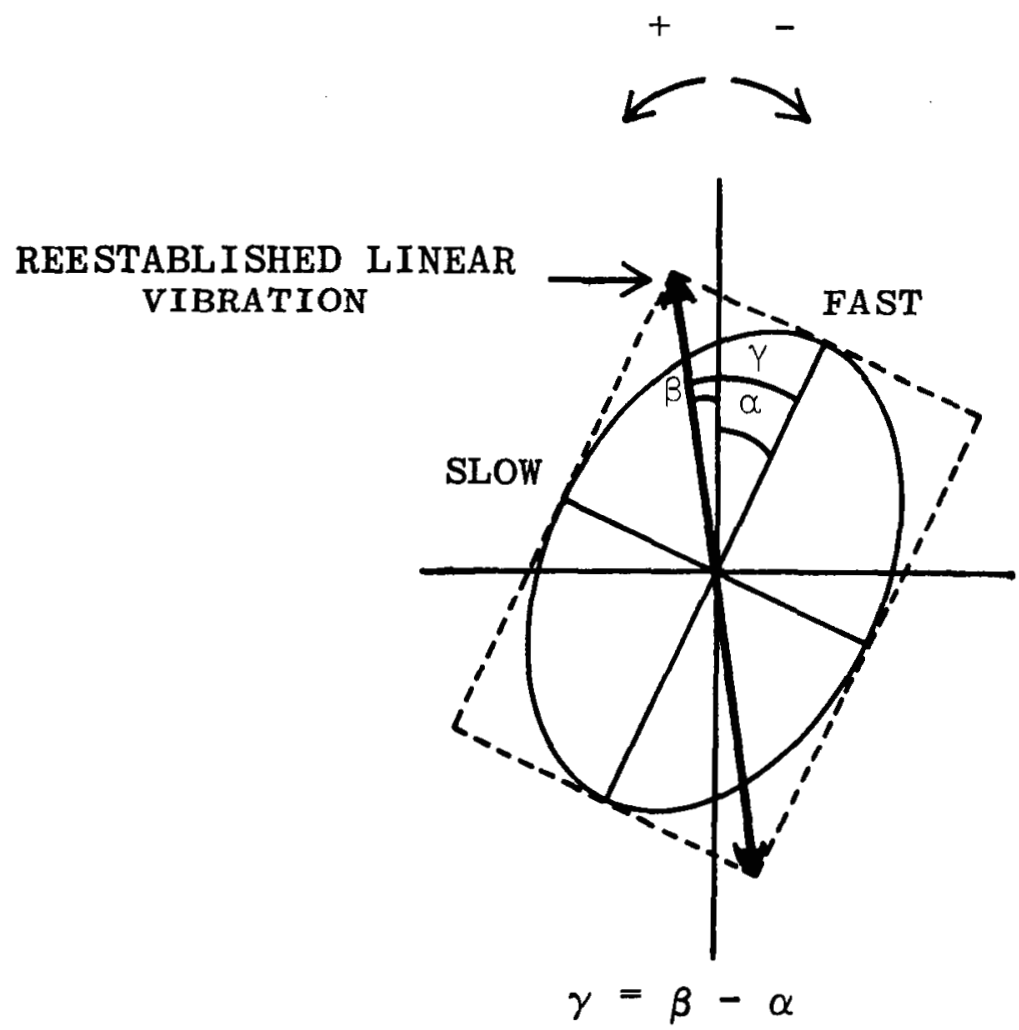

Figure 9. ELLIPTICALLY POLARIZED LIGH'T

To determine the complex index of refraction of the clean surface, $\left(\hat{\mathrm{n}}_{3}\right.$ $\left.=\hat{n}_{s_{1}}-\hat{n}_{32} i\right)$, an experimental value of $\Psi^{\prime}$ and $\Delta^{\prime}$ are determined for a film free surface. The following equations are then used to calculate $\hat{\mathrm{n}}_{3}:$

(11) Vasicek, A., Ellipsometry in the Measurement of Surfaces and Thin Films, E. Passaglia, R. R. Stromberg, and J. Kruger, ed., Washington, D. C., Natl. Bur. Std., Miscellaneous Publication 256, pp 26-28. 


$$
\mathrm{n}^{2}=\mathrm{n}_{1}^{2} \sin ^{2} \varphi_{1}\left[1+\frac{\tan ^{2} \varphi_{1} \cos ^{2} 2 \Psi^{\prime}}{\left(1+\sin 2 \Psi^{\prime} \cos \Delta^{\prime}\right)^{2}}\right]
$$

$$
k=\frac{n_{1} \tan \varphi_{1} \sin \varphi_{1} \sin 2 \Psi^{\prime} \sin \Delta^{\prime}}{1+\sin 2 \Psi^{\prime} \cos \Delta^{\prime}}
$$

$$
\cos ^{2} \varphi_{3}^{\prime}=1-\frac{n_{1}^{2} \sin ^{2} \varphi_{1}}{n^{2}}
$$

$$
\hat{\mathrm{n}}_{3}^{2}=\mathrm{n}^{2}-\mathrm{k}^{2}-\mathrm{i} 2 \mathrm{nk} \cos \varphi_{3}^{\prime}
$$

$$
n_{3 I}=\left[\frac{\left(n^{2}-k^{2}\right)+\left\{\left(n^{2}-k^{2}\right)^{2}+4 n^{2} k^{2} \cos ^{2} \varphi_{3}^{\prime}\right\}^{\frac{1}{2}}}{2}\right]^{\frac{1}{2}},
$$

$$
n_{3 a}=\left[\frac{-\left(n^{2}-k^{2}\right)+\left\{\left(n^{2}-k^{2}\right)^{2}+4 n^{2} k^{2} \cos ^{2} \varphi_{3}^{\prime}\right\}^{\frac{1}{2}}}{2}\right]^{\frac{1}{2}} .
$$

$$
\begin{aligned}
& n_{1}=\text { index of refraction of first medium } \\
& \varphi_{1}=\text { angle of incidence }
\end{aligned}
$$$$
\omega^{\prime}=\text { angle of refraction in the metal }
$$

Prime values indicate a clean surface

For the theoretical calculation of $\Psi^{\prime} s$ and $\Delta^{\prime} s$, the exact Drude equations for the reflection coefficients for a film covered surface are used $(9)$ and are given by the equations:

$$
\begin{aligned}
& \hat{\mathrm{R}}_{\mathrm{p}}=\frac{\hat{\mathrm{r}}_{1 \Omega} \mathrm{p}+\hat{\mathrm{r}}_{23} \mathrm{p} \exp (-2 i \hat{\delta})}{1+\hat{\mathrm{r}}_{12} \hat{\mathrm{p}}_{2 s} \mathrm{p} \exp (-2 i \hat{\delta})}, \\
& \hat{\mathrm{R}}_{\mathrm{n}}=\frac{\hat{\mathrm{r}}_{12} \mathrm{n}+\hat{\mathrm{r}}_{3 s} \mathrm{n} \exp (-2 i \hat{\delta})}{1+\hat{\mathrm{r}}_{1 z} \hat{\mathrm{r}}_{23}{ }^{\mathrm{n}} \exp (-2 i \hat{\delta})}
\end{aligned}
$$

(9) Theroux, P., and Plumb, R. C., loc. cit., p 13. 


\section{RFP-674}

The components of light parallel and normal to the plane of incidence are represented by $p$ and $n$, respectively. The $r$ 's are the complex Fresnel coefficients and are given by the equations:

$$
\begin{aligned}
& \hat{r}_{\alpha \beta}^{p}=\frac{\hat{n}_{\beta} \cos \hat{\varphi}_{\alpha}-\hat{n}_{\alpha} \cos \hat{\varphi}_{\beta}}{\hat{n}_{\beta} \cos \hat{\varphi}_{\alpha}+\hat{n}_{\alpha} \cos \hat{\varphi}_{\beta}}, \\
& \hat{r}_{\alpha \beta}{ }^{n}=\frac{\hat{n}_{\alpha} \cos \hat{\varphi}_{\alpha}-\hat{n}_{\beta} \cos \hat{\varphi}_{\beta}}{\hat{n}_{\alpha} \cos \hat{\varphi}_{\alpha}+\hat{n}_{\beta} \cos \hat{\varphi}_{\beta}},
\end{aligned}
$$

where $\alpha=1$ or 2 , and $\beta=2$ or 3 .

The subscripts 1,2 , and 3 refer to the surrounding medium, film, and substrate, respectively. The $n^{\prime}$ s are the complex indices of refraction and the $\varphi$ 's are the complex angles of propagation in the medium. The n's and $\varphi$ 's are related by Snell's law:

$$
\hat{\mathrm{n}}_{\alpha} \sin \hat{\varphi}_{\alpha}=\hat{\mathrm{n}}_{\beta} \sin \hat{\varphi}_{\beta}
$$

Thickness is related by the expression:

$$
\hat{\delta}=\frac{2 \pi}{\lambda} \hat{n}_{z} \cos \hat{\varphi}_{z} d
$$

where $d$ is film thickness in angstroms and $\lambda$ is wavelength of light used in angstroms. The fundamental equation of ellipsometry is:

$$
\frac{R_{p}}{R_{n}}=\tan \psi e^{i \Delta} .
$$


To determine solutions for $\Psi$ and $\Delta$, various values of $n_{2}$ are used with parious values of $d$. Equation (16) is solved for $\Psi$ and $\Delta$ by separation into its real and imaginary parts; then, a theoretical curve of $\Delta$ versus $\Psi$ is matched with the experimental curve to determine $n_{2}$ and $d$. 
RFP- 674 